\title{
Hilbert Space Models Commodity Exchanges
}

\author{
Paul Cockshott \\ Dept Computing Science University of Glasgow \\ wpc@dcs.gla.ac.uk
}

\begin{abstract}
It is argued that the vector space measures used to measure closeness of market prices to predictors for market prices are invalid because of the observed metric of commodity space. An alternative representation in Hilbert space within which such measures do apply is proposed. It is shown that commodity exchanges can be modeled by the application of unitary operators to this space.
\end{abstract}

\section{$1 \quad$ Linear Price Models}

The context of this paper is the empirical testing of linear models of economic activity. Whilst these originated in an informal way in the work of Adam Smith and Quesney, and were partially formalised by Marx in volume 3 of Capital, an adequate formal treatment had to wait for von Neuman 21. and Kantorovich $[9$. Both von Neumann and Kantorovich were mathematicians rather than economists. Their contributions to economics were just one part of a variety of research achievements. In both cases this included stints working on early nuclear weapons programs, for the US and USSR [15] respectively. At least in von Neumann's case the connection of his economic work to atomic physics was more than incidental. One of his great achievements was his mathematical formalization of quantum mechanics 22 which unified the matrix mechanics of Heisenberg with the wave mechanics of Schrodinger. His work on quantum mechanics coincided with the first draft of his economic growth model[21] given as a lecture in Princeton in 1932. In both fields he employs vector spaces and matrix operators over vector spaces, complex vector spaces in the quantum mechanical case, and real vector spaces in the growth model. Kurz and Salvadori [1] argue that his growth model has to be seen as a response to the prior work of the mathematician Remak[14], who worked on 'superposed prices'.

Remak then constructs 'superposed prices' for an economic system in stationary conditions in which there are as many single-product processes of production as there are products, and each process or product is represented by a different 'person' or rather activity or industry. The amounts of the different commodities acquired by a person over a certain period of time in exchange for his or her own product are of course the amounts needed as means of production to produce this product and the amounts of consumption goods in support of the person (and 
his or her family), given the levels of sustenance. With an appropriate choice of units, the resulting system of 'superposed prices' can be written as

$$
p^{T}=p^{T} C
$$

where $C$ is the augmented matrix of inputs per unit of output, and $p$ is the vector of exchange ratios. Discussing system Remak arrived at the conclusion that there is a solution to it, which is semipositive and unique except for a scale factor. The system refers to a kind of ideal economy with independent producers, no wage labour and hence no profits. However, in Remak's view it can also be interpreted as a socialist economic system [1].

With Remak the mathematical links to the then emerging matrix mechanics are striking - the language of superposition, the use of a unitary matrix operator $C$ analogous to the Hermitian operators in quantum mechanic: 1 . Remak shows for the first time how, starting from an in-natura description of the conditions of production, one can derive an equilibrium system of prices. This implies that the in-natura system contains the information necessary for the prices and that the prices are a projection of the in-natura system onto a lower dimensional space 2 . If that is the case, then any calculations that can be done with the information in the reduced system $p$ could in principle be done, by some other algorithmic procedure starting from $C$. Remak expresses confidence that with the development of electric calculating machines, the required large systems of linear equations will be solvable.

The weakness of Remak's analysis is that it was limited to an economy in steady state.Von Neumann took the analysis on in two distinct ways:

1. He models an economy in growth, not a static economy. He assumes an economy in uniform proportionate growth. He explicitly abjures considering the effects of restricted natural resources or labour supply, assuming instead that the labour supply can be extended to accommodate growth. This is perhaps not unrealistic as a picture of an economy undergoing rapid industrialization ( for instance Soviet Russia at the time he was writing ).

2. He allows for there to be multiple techniques to produce any given good Remak only allowed one. These different possible productive techniques use different mixtures of inputs, and only some of them will be viable.

von Neumann again uses the idea of a technology matrix introduced by Remak, but now splits it into two matrices $A$ which represents the goods con-

\footnotetext{
${ }^{1}$ Like the Hermitian operators in quantum mechanics, Remak's production operator is unitary because $p$ is an eigen vector of $C$ and $|p|$ is unchanged under the operation.

${ }^{2}$ Suppose $C$ is an $n \times n$ square matrix, and $p$ an $n$ dimensional vector. By applying Iverson's reshaping 87 operator $\rho$, we can map $C$ to a vector of length $n^{2}$ thus $\mathbf{c} \leftarrow(n \times n) \rho C$, and we thus see that the price system, having $n$ dimensions involves a massive dimension reduction from the $n^{2}$ dimensional vector $\mathbf{c}$.
} 
sumed in production, and $B$ which represents the goods produced. So $a_{i j}$ is the amount of the $j$ th product used in production process $i$, and $b_{i j}$ the amount of product $j$ produced in process $i$. This formulation allows for joint production, and he says that the depreciation of capital goods can be modeled in this way, a production process uses up new machines and produces as a side effect older, worn machines. The number of processes does not need to equal the number of distinct product types, so we are not necessarily dealing with square matrices.

Like Remak he assumes that there exists a price vector $y$ but also an intensity vector $x$ which measures the intensity with which any given production process is operated. Later the same formulation was used by Kantorovich. Two remaining variables $\beta$ and $\alpha$ measure the interest rate and the rate of growth of the economy respectively.

He makes two additional assumptions. First is that there are 'no profits', by which he means that all production processes with positive intensity return exactly the rate of interest. He only counts as profit, earning a return above the rate of interest. This also means that no processes are run at a loss ( returning less than $\beta$ ). His second assumption is that any product produced in excessive quantity has a zero price.

He goes on to show that in this system there is an equilibrium state in which there is a unique growth rate $\alpha=\beta$ and definite set of intensities and prices. The intensities and prices are simultaneously determined.

Von Neumann's work was influential in economic theory, spawing a number of similar models, probably the most famous of which was Sraffa's [18.

At the time that von Neumann was writing, despite Remak's optimism, it was not possible to empirically test different linear theories of prices because of the problem of collecting the necessary data, and the problem of solving large matrix equations. From the 1950s onwards though, the empirical problem of obtaining the $A$ and $B$ matrices was reduced by the publication of national input output tables. Since the ready availability of computers emprical testing became possible.

In 1983 Farjoun and Machover published a seminal work applying statistical mechanics to the dynamics of capitalist economies 6 . One of the predictions of their book was that what they called vertically integrated labour coefficients would be good predictors for market prices. One can view their price model as being similar to that of Remak with added thermal noise. Their predictions have largely been born out by subsequent empirical studies [2]19]12]3|13|17|4, though there have been isolated studies questioning this 10/20]. There has been some controversy as to what metric was appropriate for determining the closeness of market prices to integrated labour coefficients. In the recent literature discussing this $10[13[16 \mid 20$ it has been taken as given that the use of vector space measures is appropriate. For example one measure proposed has been to determine the angle between two price vectors. I wish to point out that this approach is questionable. 


\section{The Vector Space Problem}

Vector spaces are a subclass of metric space. A metric space is characterized by a positive real valued metric function $\delta(p, q)$ giving the distance between two points, $p, q$. This distance function must satisfy the triangle inequality $\delta(p, q) \leq$ $\delta(p, r)+\delta(q, r)$. In vector spaces this metric takes the form:

$$
\delta(\mathbf{p}, \mathbf{q})=\sqrt{\sum\left(p_{i}-q_{i}\right)^{2}}
$$

We have argued elsewhere 1 that the metric of commodity space does not take this form. Let us recapitulate the argument.

Conjecture 1. Commodity space is a vector space.

Assume that we have a commodity space made up of two commodities, gold and corn and that 1 oz gold exchanges for 100 bushels of corn. We can represent any agent's holding of the two commodities by a 2 dimensional vector $\mathbf{c}$ with $c_{0}$ being their gold holding and $c_{1}$ being their corn holding. Given the exchange ratio above, we can assume that $(1,0)$ and $(0,100)$ are points of equal worth and assuming that commodity space is a vector space thus

$$
\delta((0,0),(1,0))=\delta((0,0),(0,100))
$$

This obviously does not meet equation 1 but if we re-normalise the corn axis by dividing by its price in gold, we get a metric

$$
\delta_{c}(\mathbf{p}, \mathbf{q})=\sqrt{\left(p_{0}-q_{0}\right)^{2}+\left(\frac{p_{1}-q_{1}}{100}\right)^{2}}
$$

which meets the equation we want for our two extreme points:

$$
\delta_{c}((0,0),(1,0))=\delta_{c}((0,0),(0,1))
$$

If this is our metric, then we can define a set of commodity holdings that are the same distance from the origin as holding 1 oz of gold. Let us term this $U$ the unit circle in commodity space:

$$
U=\left\{a \in U: \delta_{c}((0,0), a)=1\right\}
$$

Since these points are equidistant from the origin, where the agent holds nothing, they must be positions of equal worth, and that movements along this path must not alter the net worth of the agent. Let us consider a point on $U$, where the agent holds $\frac{1}{\sqrt{2}}$ oz gold and $\frac{100}{\sqrt{2}}$ bushels of corn.

Would this in reality be a point of equal worth to holding 1 oz of gold?

No, since the agent could trade their $\frac{100}{\sqrt{2}}$ bushels of corn for a further $\frac{1}{\sqrt{2}} \mathrm{oz}$ gold and end up with $\sqrt{2}$ oz $>1$ oz of gold. Thus there exists a point on $U$ that is not equidistant from the origin, hence equation 3 can not be the form of the metric of commodity space and thus conjecture 1 falls, and commodity space is not a vector space. 


\section{The Metric of Commodity Space}

The metric actually observed in the space of bundles of commodities is:

$$
\delta_{b}(\mathbf{p}, \mathbf{q})=\left|\sum \alpha_{i}\left[p_{i}-q_{i}\right]\right|
$$

where $\mathbf{p}, \mathbf{q}$ are vectors of commodities, and $\alpha_{i}$ are relative values. The 'unit circle' in this space actually corresponds to a pair of parallel hyperplanes on above and one below the origin. One such hyperplane is the set of all commodity combinations of positive value 1 and the other, the set of all commodity combinations of value -1 . The latter corresponds to agents with negative worth, i.e., net debtors.

Because of its metric, this space is not a vector space and it is questionable whether measures of similarity based on vector space metrics are appropriate for it. However it is possible to posit an underlying linear vector space of which commodity space is a representation.

\section{Commodity Amplitude Space}

We will now develop the concept of an underlying space, commodity amplitude space, which can model commodity exchanges and the formation of debt. Unlike commodity space itself, this space, is a true vector space whose evolution can be modeled by the application of linear operators. The relationship between commodity amplitude space and observed holdings of commodities by agents is analogous to that between amplitudes and observables in quantum theory.

Let us consider a system of $n$ agents and $m$ commodities, and represent the state of this system at an instance in time by a complex matrix $\mathbf{A}$, where $a_{i j}$ represents the amplitude of agent $i$ in commodity $j$. The actual value of the holding of commodity $j$ by agent $i$, we denote by $h_{i j}$ an element of the holding matrix $\mathbf{H}$. This is related to $a_{i j}$ by the equation $a_{i j}=\sqrt{h_{i j}}$.

\subsection{Commodity Exchanges}

We can represent the process of commodity exchange by the application of rotation operators to A. An agent can change the amplitudes of their holdings of different commodities by a rotation in amplitude space. Thus an initial amplitude of 1 in gold space by an agent can be transformed into an amplitude of 1 in corn space by a rotation of $\frac{\pi}{2}$. Borrowing Dirac notation we can write these as 1 - gold $\dot{i}$, and 1 - $\operatorname{corn} \dot{i}$. A rotation of $\frac{\pi}{4}$ on the other hand would move an agent from a pure state 1 -gold $i$ to a superposition of states $\frac{1}{\sqrt{2}} \mid$ gold $>+\frac{1}{\sqrt{2}} \mid \operatorname{corn} i$. Unlike rotation operators in commodity space this is value conserving since on squaring we find their assets are now $\frac{1}{2}$ gold $+\frac{1}{2}$ corn.

The second conservation law that has to be maintained in exchange is conservation of the value of each individual commodity, there must be no more or less of any commodity after the exchange than there was before. This can be 
modeled by constraining the evolution operators on commodity amplitude space to be such that they simultaneously perform a rotation on rows and columns of the matrix A.

Suppose we start in state:

$$
\mathbf{A}=\left[\begin{array}{ll}
1 & 0 \\
0 & 2
\end{array}\right], \mathbf{H}=\left[\begin{array}{ll}
1 & 0 \\
0 & 4
\end{array}\right]
$$

Where agent zero has 1 of gold and no corn, and agent one has no gold and 4 of corn. We can model the purchase of 1 of corn by agent zero from agent one by the evolution of $\mathbf{A}$ to:

$$
\mathbf{A 2}=\left[\begin{array}{ll}
0 & 1 \\
1 & \sqrt{3}
\end{array}\right]
$$

which corresponds to final holdings of:

$$
\mathbf{H 2}=\left[\begin{array}{ll}
0 & 1 \\
1 & 3
\end{array}\right]
$$

Note that the operation on amplitude space is a length preserving rotation on both the rows and the columns. The lengths of the row zero and column zero in $\mathbf{A 2}$ are 1 the lengths of row and column one is 2 just as it was for A. This operation can be effected by the application of an appropriate rotation matrix so that $\mathbf{A} 2=$ M.A. A matrix which produces this particular set of rotations is:

$$
\mathbf{M}=\left[\begin{array}{ll}
0 & \frac{1}{2} \\
1 & \frac{\sqrt{3}}{2}
\end{array}\right]
$$

\subsection{Price Changes}

Price movements are equivalent to the application of scaling operations which can be modeled by the application of diagonal matrices. Thus a $50 \%$ fall in the price of corn in our model would be represented by the application of the matrix 10 $0 \frac{1}{\sqrt{2}}$ to the current commodity amplitude matrix. Scaling operations are not length preserving.

The reason for this is that if there is a change in prices an agent holding a vector of physical commodities $\mathbf{b}$ will find that for most arbitrarily chosen commodity vectors $\mathbf{c}$, the quantity of $\mathbf{c}$ that they can exchange $\mathbf{b}$ for will have changed. The length preserving rotations that we have assumed up to now have amounted to assuming that there is no possibility of changing ones net worth by commodity exchanges at a given set of relative prices. If the prices change over time this is no longer the case, hence the introduction of non-conservative operations. 


\subsection{Modeling Debt}

We specified in section 4 that the amplitude matrix must be complex valued. This is required to model debt. Suppose that starting from holdings $\mathbf{H}$ agent zero buys 2 of corn from agent one. Since agent zero only has 1 of gold to pay for it, the transaction leaves the following holdings:

$\begin{array}{ccc}\text { Agent gold corn } \\ 0 & -1 & 2 \\ 1 & 2 & 2\end{array}$

The corresponding amplitude matrix is

$$
\mathbf{A 3}=\left[\begin{array}{cc}
i & \sqrt{2} \\
\sqrt{2} & \sqrt{2}
\end{array}\right]
$$

It it interesting that this too is the result of applying a unitary rotation operator to the original amplitude vector since the length of row zero $\left|\mathbf{A} \mathbf{3}_{0}\right|=$ $i^{2}+(\sqrt{2})^{2}=1$, likewise the lengths of all other rows and columns are preserved. The linear operator required to create debts has itself to be complex valued, thus if $\mathbf{A 3}=\mathbf{N A}$ we have

$$
\mathbf{N}=\left[\begin{array}{cc}
i & \frac{1}{\sqrt{2}} \\
\sqrt{2} & \frac{1}{\sqrt{2}}
\end{array}\right]
$$

Note that the operators here are not Hermitian. This would appear to preclude the interesting possibility of simulating commodity exchanges on a future quantum computer [5], though there may be renormalization techniques that could be applied to get over this problem.

\section{Implications for Similarity Measures}

Steedman [20] has proposed that a suitable criterion for assessing similarity of values to market prices is the angle between market price and value vectors, with small angles indicating closeness. If $\mathbf{m}, \mathbf{v}$ are market price and value vectors respectively, the angle between them is given by:

\section{$\operatorname{ArcCos}(\overrightarrow{\mathbf{m}} \cdot \overrightarrow{\mathbf{v}})$}

where $\mathbf{v}$ denotes the normalized value vector given by $\mathbf{v}=\frac{\mathbf{v}}{|\mathbf{v}|}$.

If the argument in section 2 is accepted, we should consider using angles between price and value amplitude vectors instead. If we denote the normalized vectors in amplitude space by $\mathbf{m}_{\boldsymbol{a}}$ and $\mathbf{v}_{\boldsymbol{a}}$, then the amplitude space angles are given by:

$$
\operatorname{ArcCos}\left(\overrightarrow{\mathbf{m}_{a}} \cdot \overrightarrow{\mathbf{v}_{\mathbf{a}}}\right)
$$

where $\mathbf{x}^{*}$ is the conjugate of $\mathbf{x}$.

What will be the properties of this measure?

In general it will show smaller angles between vectors. For example suppose we have 3 commodities iron, corn, cotton as follows: 


\begin{tabular}{rcccc|}
\hline & \multicolumn{3}{c|}{ amplitudes } \\
value price value price \\
corn & 1 & 1 & 1 & 1 \\
iron & 3 & 2 & $\sqrt{3}$ & $\sqrt{2}$ \\
cotton & 1 & 2 & 1 & $\sqrt{2}$ \\
\cline { 2 - 4 } angle & $30.2^{\circ}$ & \multicolumn{2}{|c}{$13.4^{\circ}$} \\
\cline { 2 - 4 }
\end{tabular}

The fact that smaller angles are shown would be or little significance if the relative sizes of angles in the two spaces was the same. But this need not be the case. Consider the following example:

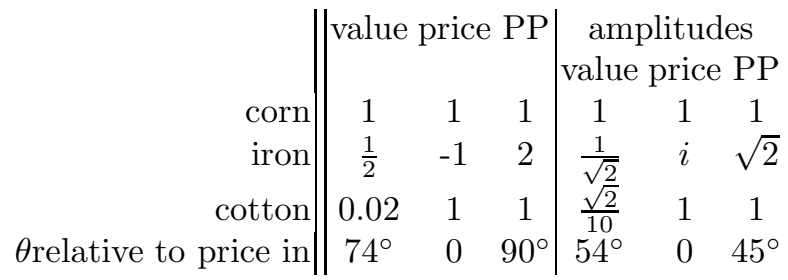

Here we are comparing three hypothetical vectors of values, prices and prices of production $(\mathrm{PP})$. If we treated commodity value space as a vector space, then prices of production would be orthogonal to market prices, whereas in amplitude space they are at $45^{\circ}$ to market prices. In this example, when commodity space is treated as a vector space, values appear closer to market prices than do prices of production. When the assumption that commodity space is a vector space is dropped, then prices of production are closer to market prices.

\section{Conclusion}

We have argued that commodity space can not be directly modeled by a vector space, because of the metric it observes, but that it can be treated as the real valued representation or an underlying vector space. This complex vector space we have, following physics terminology, termed commodity amplitude space. Observed holdings of commodities and money by agents are the squares of corresponding commodity amplitudes. Commodity exchange relations, including the formation of commercial debt can be modeled by unitary rotation matrices operating on this amplitude space. The conceptual model presented borrows extensively from quantum formalism.

It is thus at least arguable that the empirical relation between market prices and labour values should be measured by the angles between their corresponding vectors in commodity amplitude space. The latter space, unlike commodity space, is a linear vector space within which angles of rotation have a clear meaning.

\section{References}

1. Cockshott, P., Cottrell, A.: Value's Law, Value's Metric, research report University of Strathclyde, Dept. of Computer Science (1994); Reprinted in: Freeman, A., Kliman, A., Wells, J. (eds.): The New Value Controversy and the Foundations of Economics, Edward Elgar, Cheltenham (2004) 
2. Cockshott, W.P., Cottrell, A.: Labour-Time Versus Alternative Value Bases: A Research Note. Cambridge Journal of Economics 27, 749-754 (1997)

3. Cockshott, W.P., Cottrell, A.: A note on the organic composition of capital and profit rates. Cambridge Journal of Economics 21, 545-549 (2003)

4. Cockshott, W.P., Cottrell, A., Michaelson, G.: Testing Marx: Some New Results from UK Data. Capital and Class 55, 103-129 (Spring 1995)

5. DiVincenzo, D.: Quantum Computation. Science 270, 255-261

6. Farjoun, E., Machover, M.: The laws of Chaos. Verso (1983)

7. Iverson, K.: A programming language. Wiley, New York (1966)

8. Iverson, K.: Notation as a tool of thought. In: ACM Turing award lectures, p. 1979. ACM, New York (2007)

9. Kantorovich, L.V.: Mathematical Methods of Organizing and Planning Production. Management Science 6(4), 366-422 (1960)

10. Kliman, A.: The Law of Value and Laws of Statistics: Sectoral Values and Prices in the US Economy, 1977-1997. Cambridge Journal of Economics 26, 299-311 (2002)

11. Kurz, H.D., Salvadori, N.: Von Neumann's Growth Model and the 'Classical' Tradition. Understanding "classical" Economics: Studies in Long-Period Theory (1998)

12. Ochoa, E.M.: Values, prices, and wage-profit curves in the US economy. Cambridge Journal of Economics 13(3), 413-429 (1989)

13. Petrovic, P.: The deviation of production prices from labour values: some methodology and empirical evidence. Cambridge Journal of Economics 11(3), 197-210 (1987)

14. Remak, R.: Kann die Volkswirtschaftslehre eine exakte Wissenschaft werden. Jahrbücher für Nationalökonomie und Statistik 131, 703-735 (1929)

15. Ryabev, L.D., Smirnov, Y.N.: The Atomic Project, Science, and the Atomic Industry. Atomic Energy 99(2), 519-527 (2005)

16. Shaik, A.: The Empirical Strength of the Labour Theory of Value. In: Bellafiore, R. (ed.) Marxian Economics. A reapraisal Essays on Volume III of Capital. Profits, Prices and Dynamics, pp. 225-251. St. Martins Press, New York (1998)

17. Shaikh, A.: The transformation from Marx to Sraffa. In: Freeman, A., Mandel, E. (eds.) Ricardo, Marx, Sraffa, pp. 43-84. Verso, London (1984)

18. Sraffa, P.: Production of commodities by means of commodities. Cambridge University Press, Cambridge (1960)

19. Valle Baeza, A.: Correspondence between labor values and prices: a new approach. Review of Radical Political Economics 26(2), 57-66 (1994)

20. Steedman, I., Tomkins, J.: On measuring the deviation of prices from values. Cambridge Journal of Economics 22, 359-369 (1998)

21. von Neumann, J.: A Model of General Economic Equilibrium. Review of Economic Studies 13(33), 1-9 (1945)

22. von Neumann, J.: Mathematical Foundations of Quantum Mechanics, Engl. transl. of the 1931 German edition by Beyer, R.T. (1955) 